\title{
REMEDIACIÓN ELECTROCINÉTICA DE UN SUELO REAL CONTAMINADO CON PLOMO ASISTIDA CON LA ADICIÓN DE UN AGENTE COMPLEJANTE
}

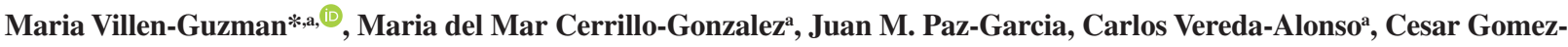 \\ Lahoz $^{\text {a }}$ Jose M. Rodriguez-Maroto ${ }^{a}$ \\ aDepartamento de Ingeniería Química, Universidad de Málaga, Campus de Teatinos, 29071 - Málaga, España
}

Recebido em 11/03/2020; aceito em 01/06/2020; publicado na web em 08/07/2020

\begin{abstract}
ENHANCED ELECTROKINETIC REMEDIATION OF A REAL LEAD-CONTAMINATED SOIL BY CHELATING AGENT ADDITION. The use of ethylenediaminetetraacetic acid disodium salt ( $\mathrm{Na}_{2}$ EDTA) as a chelating agent presents ideal characteristics to be used for the removal of lead from a contaminated site. Batch experiments together with a simple local equilibrium model were carried out to evaluate the role of EDTA as enhancing agent in electrokinetic remediation of a Pb-contaminated soil. According to preliminary results, $\mathrm{Pb}$ removal was close to $85 \%$ after 9 hours. Hence, electrokinetic experiments were designed implementing a $\mathrm{pH}$ control system at both electrode-compartments. From first experiments, no important Pb recovery was obtained probably due to the counteracting effect of the electroosmotic and electromigration movement of Pb-species. The use of an iron anode instead of a platinum anode involved the recovery of a high percentage of $\mathrm{Pb}(80 \%)$ at the cathode. These results were associated with the behavior of the iron as a sacrificial anode. Furthermore, the formation of $\mathrm{Fe}(\mathrm{EDTA})^{-}$hinders the formation of Pb(EDTA) ${ }^{2-}$, which entails the dissolution of the contaminant as $\mathrm{Pb}^{2+}$. Therefore, the $\mathrm{Pb}$ species are transported toward the cathode by electromigration and electroosmosis. Furthermore, the competition between the oxidation of iron and the water electrolysis entailed a lower consumption of $\mathrm{NaOH}$ to control $\mathrm{pH}$ value at the anode compartment.
\end{abstract}

Keywords: electrokinetic; soil remediation; lead; complexing agent; sacrificial anode.

\section{INTRODUCCIÓN}

En la actualidad, la contaminación de suelos constituye un problema medioambiental al que se debe prestar especial atención por constituir un elemento esencial de la biosfera. A pesar de su importancia, la normativa referente a suelos contaminados se caracteriza por un desarrollo tardío respecto a otros medios como el atmosférico y el hidrosférico. Hasta 1980, como consecuencia de desastres ambientales como el de Love Canal, ${ }^{1}$ no se promulga la primera ley referente a suelos contaminados en EEUU, denominada CERCLA (Comprehensive Environmental Response, Compensation and Liability Act).

Como resultado de la intensificación de actividades industriales y mineras, los suelos se ven sometidos a altas concentraciones de metales pesados lo que constituye un serio problema para el medioambiente. ${ }^{2}$ En este punto, se debe tener en cuenta que los metales pesados presentan tendencia a ser retenidos en el suelo por diversos mecanismos como precipitación, adsorción e intercambio iónico, lo que hace más compleja su eliminación. ${ }^{3}$ Por tanto, el estudio de la movilidad de este tipo de contaminantes resulta esencial para evaluar el riesgo asociado a la presencia de metales y la viabilidad de las técnicas de recuperación del suelo. ${ }^{4,5}$ Para ello, se ha demostrado que la aplicación de métodos de extracción secuencial (Sequential Extraction Procedures, SEPs) supone una herramienta de gran utilidad. Estas técnicas consisten, básicamente, en la adición de diferentes disoluciones en tres etapas consecutivas a través de las que se obtienen diversas fracciones de metales en función de su dificultad para ser movilizados en el suelo. ${ }^{6}$ En la actualidad, el método normalizado más ampliamente aplicado a diversas matrices sólidas es el denominado "revisado BCR". ${ }^{7}$ Estas técnicas han sido ampliamente empleadas para evaluar los cambios en la movilidad de los metales tras aplicar una técnica de descontaminación. ${ }^{89}$ Así, en

*e-mail: mvillen@uma.es algunas ocasiones, se ha detectado un aumento de la movilidad del contaminante tras aplicar la técnica de remediación del suelo. ${ }^{10}$ En estos casos, a pesar de que exista una disminución de la concentración total del contaminante, el riesgo potencial del suelo tras ser tratado aumenta. Esto pone de manifiesto la utilidad de los métodos de extracción secuencial para determinar la eficiencia de la técnica de remediación.

Por otro lado, la caracterización del suelo contaminado resulta de gran relevancia para la selección de la técnica de recuperación más adecuada. Así, en el caso de suelos con una baja conductividad hidráulica no resulta viable la aplicación de técnicas convencionales basadas en el movimiento del líquido a través de la matriz sólida. ${ }^{11}$ Para este tipo de suelos, la técnica de electrodescontaminación resulta la mejor alternativa. Esta tecnología se basa en la aplicación de una corriente eléctrica de baja intensidad entre electrodos ubicados en el suelo. Como resultado de la generación de una densidad de corriente tiene lugar el transporte de las especies presentes en el suelo hacia el ánodo o el cátodo desde donde, posteriormente, son separadas. ${ }^{12,13}$ Los mecanismos implicados en el movimiento de las especies bajo una corriente eléctrica son electromigración, electroósmosis, electroforesis y difusión química. El movimiento de las especies iónicas disueltas hacia el electrodo de carga opuesta se conoce como electromigración y es uno de los mecanismos predominantes en presencia de una corriente eléctrica. Por otro lado, la electroósmosis consiste en el movimiento del líquido a través de los poros del suelo. ${ }^{14}$ Además de los mecanismos de transporte, resulta de especial relevancia la electrólisis del agua como consecuencia de la aplicación de una corriente eléctrica a un suelo saturado. ${ }^{15}$ Debido a estas reacciones, tiene lugar la producción de protones y oxígeno gaseoso en el ánodo e iones hidroxilos e hidrógeno gaseoso en el cátodo. De esta forma, el pH del suelo varía a lo largo del mismo con la aplicación de la corriente eléctrica generándose un frente de protones que viaja desde el ánodo hacia el cátodo y un frente de hidroxilos que viaja en dirección contraria. Estos cambios de $\mathrm{pH}$ influyen directamente 
en los procesos químicos que tienen lugar en el suelo. En el caso de los metales pesados, el aumento de $\mathrm{pH}$ en la zona del suelo cercana al cátodo provoca la precipitación y acumulación de las especies metálicas provocando el frenado del proceso de recuperación del suelo. Por ello, se hace necesaria la aplicación de algún método de mejora de la técnica. ${ }^{16,17}$

Uno de los métodos de mejora más empleados es la adición de ácidos, bases o sustancias complejantes. ${ }^{8,18-21}$ Por sus características, destaca el uso del ácido etildiaminotetraacético (EDTA, abreviado frecuentemente como $\mathrm{H}_{4} \mathrm{Y}$, donde $\mathrm{Y}$ hace referencia al anión EDTA $^{4-}$ ) como agente complejante. ${ }^{22-25}$ El EDTA se disocia en agua dando lugar a $\mathrm{H}_{3} \mathrm{Y}^{-}, \mathrm{H}_{2} \mathrm{Y}^{2-}$ y $\mathrm{Y}^{4-}$ con valores de $p \mathrm{Ka}$ : 2, 2.76, 6.27 y 10.95, respectivamente. La posibilidad de que cada anión EDTA ${ }^{4-}$ pueda formar complejo con un ion metálico en seis posiciones diferentes (cuatro asociadas con los grupos acetatos y dos asociadas con los dos pares de electrones libres de los átomos de nitrógeno) da lugar a la formación de complejos Metal-EDTA caracterizados por una alta estabilidad. ${ }^{26}$ Esto hace que sea un candidato ideal para ser empleado como agente de mejora en la técnica de electrodescontaminación cuando se tienen altas concentraciones de metales como el $\mathrm{Pb}$. Así, el transporte del agente complejante a través del suelo conlleva la desorción de los iones metálicos mediante la formación de nuevas especies que pueden moverse hacia los compartimentos electródicos por electromigración o electroósmosis. ${ }^{23}$ De acuerdo con Yeung et al., que aplicaron la técnica electrocinética mejorada con EDTA a una caolinita contaminada con $\mathrm{Pb}$, el papel del agente complejante permite recuperar, aproximadamente, un $90 \%$ del contaminante contenido en el suelo en el ánodo. A partir de este estudio, se comienza a evaluar la dependencia de la efectividad de la técnica mejorada con EDTA con las condiciones experimentales, destacando el valor de $\mathrm{pH}$ en el suelo por su gran influencia en los fenómenos de transporte electrocinéticos. ${ }^{27}$ Desde entonces, diferentes autores han abordado el estudio del EDTA como agente de mejora de la técnica electrocinética para diversos suelos y contaminantes. Así, Reddy et al., que aplicaron la técnica de electrodescontaminación mejorada con EDTA a una caolinita contaminada artificialmente con $\mathrm{Cd}$, Ni y Cr, obtuvieron una baja recuperación de los contaminantes. Este estudio concluyó que, posiblemente, la baja movilidad del EDTA y la dirección de su transporte por electromigración, contraria a la del flujo electroosmótico, causó la no recuperación de los contaminantes. ${ }^{28}$ Posteriormente, M. Villen et al. estudiaron los posibles efectos negativos de la corriente eléctrica en la formación de complejos metal-EDTA y evaluaron los equilibrios químicos mediante un modelo matemático. Tras llevar a cabo ensayos a escala semi-piloto, corroboraron que la dirección opuesta de los flujos por electroósmosis y electromigración era la explicación a la nula recuperación del contaminante tras aplicar la técnica electrocinética mejorada con EDTA. ${ }^{29}$

El presente trabajo persigue elucidar la problemática, reportada en bibliografía, relacionada con el uso del EDTA como agente de mejora en la aplicación de la técnica electrocinética. Se debe destacar, además, que existe un número limitado de estudios que aborden el uso de dicho agente complejante empleando suelos contaminados reales. La mayoría de estudios reportados en bibliografía trabajan con suelos modelos, como caolinita, lo cual limita la posibilidad de extrapolar los resultados obtenidos a aplicaciones de la técnica a escala de campo. Por ello, en este trabajo se aborda el estudio de la aplicación de la técnica electrocinética a un suelo real contaminado con $\mathrm{Pb}$ variando factores relevantes, tales como las condiciones de $\mathrm{pH}$ en los compartimentos electródicos, la disolución con la que se satura el medio poroso sólido y, además, se propone el uso combinado de EDTA y de un ánodo de sacrificio.

\section{EXPERIMENTAL}

\section{Caracterización del suelo y su contaminación}

Las muestras de suelo empleadas en este trabajo fueron recogidas en el distrito minero de Linares, situado al sur de España. ${ }^{30}$ Inicialmente, se procedió al secado y tamizado de las muestras para seleccionar la fracción de partículas con un tamaño inferior a $2 \mathrm{~mm}$. La determinación de las propiedades del suelo se llevó a cabo de acuerdo con los siguientes métodos normalizados: distribución de tamaño de partícula (ASTM D422-63), pH (ASTM D4972), densidad (ASTM854), capacidad de intercambio catiónico (EPA 9081), conductividad hidráulica (EPA 9100) y contenido en materia orgánica (ASTM D2974). La determinación de la cantidad de carbonatos presentes en el suelo se llevó a cabo mediante la combinación de un análisis termogravimétrico y calorimetría diferencial de barrido (Equipo TA instrument, modelo SDT Q600).

La concentración total de plomo en el suelo se determinó mediante digestión ácida asistida por microondas (Berghof) siguiendo el método normalizado EPA 3051A. Una vez extraídos los metales de la fase sólida, se analizó la concentración de plomo por absorción atómica de llama (Varian SpectrAA 1101). Con objeto de clasificar las distintas fracciones de plomo en función de su movilidad, se aplica el procedimiento normalizado BCR. ${ }^{7}$ Tanto la determinación de la concentración total de plomo como la aplicación del procedimiento normalizado BCR se llevaron a cabo antes y después de los ensayos de descontaminación realizados tanto en tanque agitado como en columna.

\section{Ensayos en tanque agitado}

Los ensayos en tanque agitado se llevaron a cabo en el sistema experimental presentado en la Figura 1. Éste consiste en un reactor con una capacidad de dos litros cerrado con una tapadera con varias bocas donde se introducen el electrodo de $\mathrm{pH}$, el agitador mecánico y un tubo para la toma de muestras. Los ensayos se llevaron a cabo añadiendo $400 \mathrm{~g}$ de suelo seco en $1.2 \mathrm{~L}$ de agua destilada. Una vez que comenzó la agitación, se realizaron adiciones manuales de $1.7 \mathrm{~g}$ de $\mathrm{Na}_{2}$ EDTA cada 5 minutos durante 9 horas. Pasado este tiempo, se añadieron $380 \mathrm{~g}$ adicionales y se dejó el sistema en agitación hasta que transcurrieron 24 horas. A lo largo del ensayo se extrajeron muestras de $10 \mathrm{~mL}$ empleando una jeringa de plástico, con una planificación temporal que permitió considerar despreciable el cambio de volumen en el reactor durante el ensayo.

\section{Ensayos electrocinéticos}

El sistema experimental en el que se llevaron a cabo los ensayos

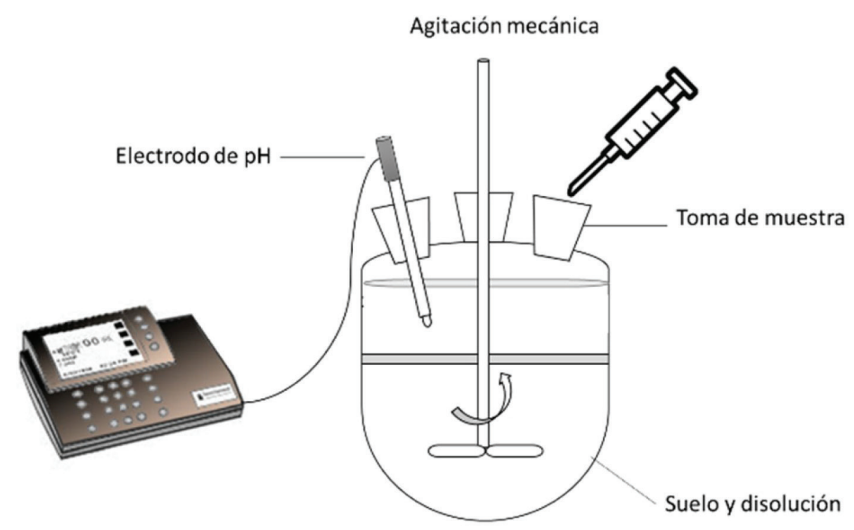

Figura 1. Sistema experimental para ensayos en tanque agitado 


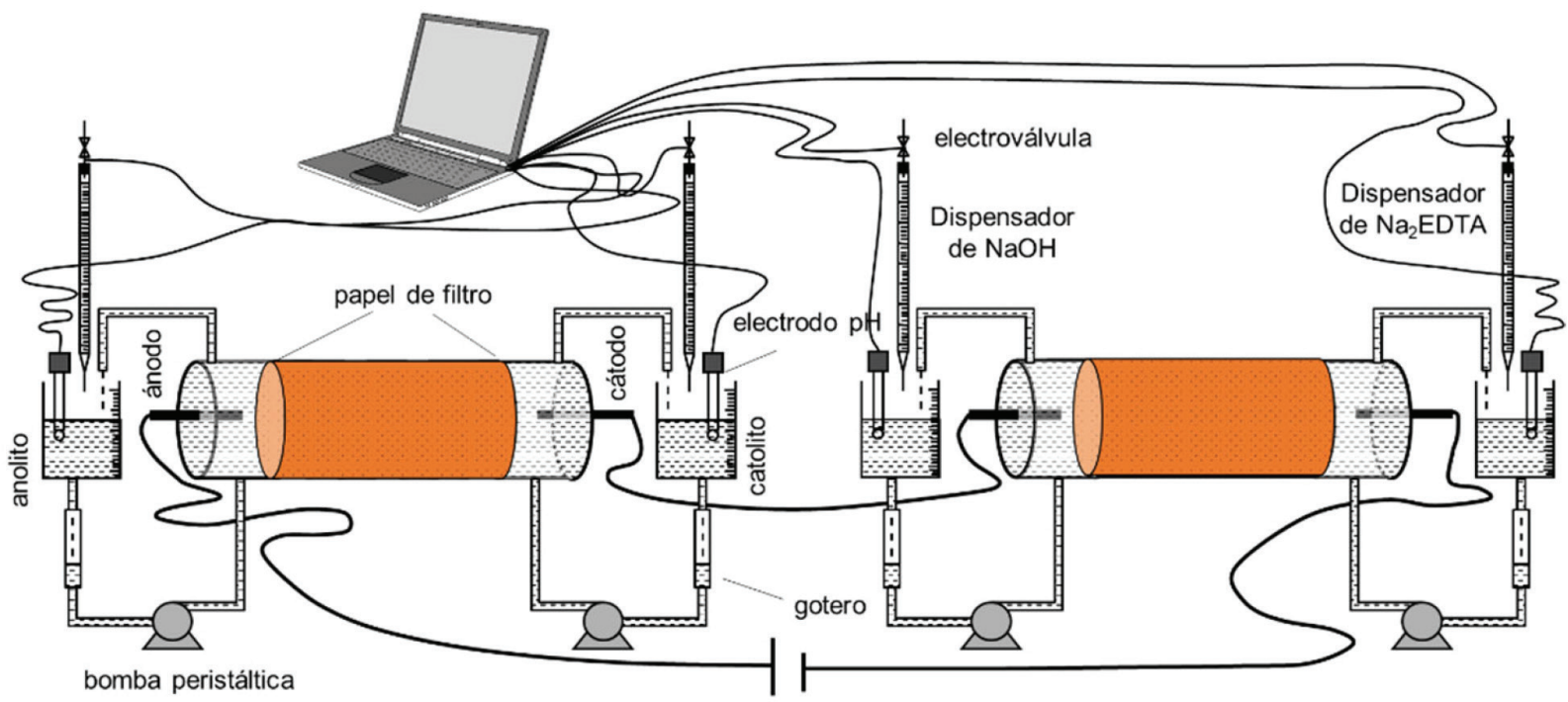

Figura 2. Sistema experimental para ensayos electrocinéticos

consiste en dos columnas de metacrilato conectadas en serie para garantizar la reproducibilidad de los ensayos por duplicado (Figura 2). A su vez, las columnas se dividen en tres secciones que facilitan el llenado y vaciado de las mismas. Una muestra de 16 gramos de suelo se introdujo en la zona central, siendo su diámetro y su longitud 2.2 y $2 \mathrm{~cm}$, respectivamente. El suelo se saturó previamente con un $32 \%$ en peso en base seca de disolución de $\mathrm{NaNO}_{3}$ o $\mathrm{Na}_{2}$ EDTA, dependiendo del ensayo, tal y como se presenta en la Tabla 1. El compartimento central que contiene el suelo se encuentra separado de los dos compartimentos electródicos mediante papel de filtro con objeto de que no exista movilización de suelo. Las dimensiones de los compartimentos electródicos, colocados a cada uno de los lados del compartimento que contiene el suelo, son de $2.2 \mathrm{~cm}$ de diámetro interno y $3 \mathrm{~cm}$ de longitud. El material del electrodo empleado como ánodo es platino o hierro, dependiendo del ensayo, y el material del cátodo es acero inoxidable. Los electrodos consisten en barras cilíndricas metálicas con una longitud y diámetro de, aproximadamente 4 y $0.5 \mathrm{~cm}$, respectivamente (Figura 3). Desde los compartimentos electródicos se recircularon $400 \mathrm{~mL}$ de disoluciones electrolíticas (Tabla 1) hacia depósitos de vidrio exteriores empleando para ello una bomba peristáltica de 4 canales. Los ensayos se llevaron a cabo con control automático de $\mathrm{pH}$ en los dos compartimentos electródicos por medio de un sistema de elaboración propia que consiste en una bureta graduada con una electroválvula instalada en la cabeza de la misma y controlada desde un ordenador desde el que se registran los valores de $\mathrm{pH}$ y la cantidad de disolución adicionada. Las disoluciones empleadas para el control de $\mathrm{pH}$ en el ánodo y cátodo, fueron $0.4 \mathrm{~mol} \mathrm{~L}^{-1}$ de $\mathrm{NaOH}$ y $0.2 \mathrm{~mol} \mathrm{~L}^{-1}$ de $\mathrm{Na}_{2}$ EDTA, respectivamente. Las condiciones experimentales seleccionadas para los diferentes ensayos realizados se detallan en la Tabla 1.

Los ensayos electrocinéticos comienzan con el encendido de la fuente de alimentación (Genesys TDK Lambda GEN 600-2.6),

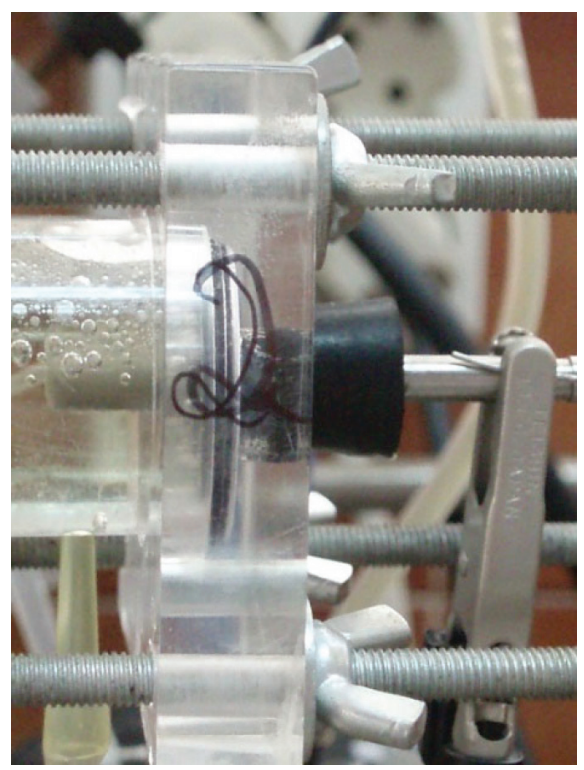

Figura 3. Detalle electrodo de Fe

conectada a los electrodos, para proporcionar un valor de densidad de corriente constante, $2 \mathrm{~mA} \mathrm{~cm}^{-2}$. Como consecuencia de la aplicación de la corriente eléctrica, las especies presentes en el suelo pueden ser transportadas hacia el ánodo o cátodo mediante diversos mecanismos, principalmente, electromigración y electroósmosis. Desde los compartimentos electródicos son posteriormente retirados y analizados. La toma de muestras de los depósitos exteriores de los electrolitos se llevó a cabo diariamente para determinar la evolución de la concentración de plomo analizada por espectrofotometría de absorción atómica. Asimismo, se determinó la caída de potencial

Tabla 1. Condiciones experimentales ensayos electrocinéticos

\begin{tabular}{|c|c|c|c|c|c|}
\hline Exp. & Disolución inicial anolito & Disolución inicial catolito & Disolución saturación del suelo & Control de $\mathrm{pH}$ & mmol e- circulados \\
\hline I & $\mathrm{NaNO}_{3} 10^{-3} \mathrm{~mol} \mathrm{~L}^{-1}$ & $\mathrm{Na}_{2}$ EDTA $10^{-3} \mathrm{~mol} \mathrm{~L}^{-1}$ & $\mathrm{NaNO}_{3} 10^{-3} \mathrm{~mol} \mathrm{~L}^{-1}$ & $\begin{array}{l}\text { Ánodo: } \mathrm{pH}=4 \\
\text { Cátodo: } \mathrm{pH}=8\end{array}$ & 112 \\
\hline II & & $\mathrm{Na}_{2}$ EDTA $10^{-3} \mathrm{~mol} \mathrm{~L}^{-1}$ & & $\begin{array}{l}\text { Ánodo: } \mathrm{pH}=4 \\
\text { Cátodo: } \mathrm{pH}=8\end{array}$ & 277 \\
\hline III & & $\mathrm{Na}_{2}$ EDTA $10^{-3} \mathrm{~mol} \mathrm{~L}^{-1}$ & & $\begin{array}{c}\text { Ánodo: } \mathrm{pH}=3.5 \\
\text { Cátodo: } \mathrm{pH}=9\end{array}$ & 277 \\
\hline
\end{tabular}


eléctrico para cada una de las columnas con objeto de analizar la evolución de la conductividad eléctrica del suelo. Con el propósito de estudiar el flujo por electroósmosis, se llevó a cabo la pesada diaria de los depósitos externos que forman parte de los compartimentos electródicos. A partir de estos datos, teniendo en cuenta que el flujo volumétrico electroosmótico $\left(J_{e o}\right)$ está relacionado con el gradiente de voltaje medio $(\Delta V / L)$ por medio de la permeabilidad electroosmótica efectiva $\left(k_{e o}^{*}\right)$ de acuerdo con la expresión: $J_{e o}=k_{e o}^{*}(\Delta V / L)$, se obtuvo el valor de $k_{e o}^{*}$ mediante el ajuste de los datos experimentales para los diferentes ensayos.

Una vez que se dieron por finalizados los ensayos, se procedió a dividir el suelo en dos secciones para determinar el valor de $\mathrm{pH}$, la concentración total de $\mathrm{Pb}$, el contenido en humedad y los cambios de movilidad del $\mathrm{Pb}$ mediante el método BCR. Las secciones 1 y 2 de suelo se corresponden, respectivamente, con las zonas de suelos cercanas al ánodo y cátodo.

\section{RESULTADOS Y DISCUSIÓN}

\section{Caracterización del suelo y su contaminación}

Las propiedades más relevantes del suelo estudiado se presentan en la Tabla 2 y se han discutido con mayor detalle en trabajos previos. ${ }^{8,31}$

Tabla 2. Condiciones experimentales ensayos electrocinéticos

\begin{tabular}{lc}
\hline Distribución de tamaño de partícula $(\%)$ & Arena: 22 \\
& Limo: 44 \\
Arcilla: 34 & $9.28 \pm 0.01$ \\
$\mathrm{pH}($ Agua destilada) $(1 / 1 \mathrm{w} / \mathrm{w})$ & $16.4 \pm 1.0$ \\
$\mathrm{CEC}\left(\mathrm{cmol} \cdot \mathrm{kg}^{-1}\right)$ & $10^{-11} \mathrm{a} 10^{-12}$ \\
Conductividad Hidráulica $\left(\mathrm{m} \cdot \mathrm{s}^{-1}\right)$ & $1.86 \pm 0.18$ \\
Materia orgánica (base seca) $(\%)$ & 2.23 \\
Densidad del suelo seco $\left(\mathrm{g} \mathrm{cm}{ }^{-3}\right)$ & $51.0 \pm 1.9$ \\
Porosidad $(\%)$ & $<2$ \\
Humedad $(\%)$ & $85000 \pm 1900$ \\
$\mathrm{CO}_{3}^{2-}\left(\mathrm{mg} \cdot \mathrm{kg}^{-1}\right)$ &
\end{tabular}

De estos resultados se concluye que se trata de un suelo francoarcilloso, con un valor de pH básico, capacidad de intercambio iónica media-baja, una conductividad hidráulica baja, bajo contenido de materia orgánica y una importante cantidad de carbonatos. La baja conductividad hidráulica del suelo pone de manifiesto la idoneidad de la aplicación de la técnica electrocinética para la recuperación del mismo. Del análisis del contenido total de metales en el suelo (Tabla 3), destacó una alta concentración de $\mathrm{Pb}, 45200 \pm 700 \mathrm{mg} \mathrm{kg}{ }^{-1}$, valor que se encuentra muy por encima del nivel genérico de referencia para uso urbano del suelo, $2750 \mathrm{mg} \mathrm{kg}^{-1}$, de acuerdo a la normativa autonómica de la zona donde se encuentra el suelo. ${ }^{32}$ Esto representa un alto riesgo medioambiental y destaca la necesidad de llevar a cabo una descontaminación del suelo.

Respecto a los resultados obtenidos aplicando el método de extracción secuencial (BCR) (Figura 4), destacó una importante concentración de $\mathrm{Pb}$ en la fracción soluble en ácido débil (WAS, Weak Acid Soluble). Se debe matizar que el metal asociado a esta fracción se caracteriza por presentar una alta movilidad y, por tanto, conlleva un alto riesgo para el medioambiente. La fracción denominada reducible (RED) representó un $35.1 \%$ del total y se asocia con las especies del metal fijadas sobre óxidos de hierro y manganeso..$^{33} \mathrm{Las}$ proporciones de $\mathrm{Pb}$ asociadas con la fase oxidable (OXI), relacionada
Tabla 3. Concentración total de metales en el suelo contaminado obtenidos mediante digestión ácida y análisis mediante absorción atómica

\begin{tabular}{cc}
\hline Metal & Concentración total (base seca) $\left(\mathrm{mg} \mathrm{kg}^{-1}\right)$ \\
\hline $\mathrm{Pb}$ & $45200 \pm 700$ \\
$\mathrm{Mn}$ & $2300 \pm 200$ \\
$\mathrm{Cu}$ & $530 \pm 16$ \\
$\mathrm{Ca}$ & $34000 \pm 2000$ \\
$\mathrm{Mg}$ & $6000 \pm 400$ \\
$\mathrm{Fe}$ & $37000 \pm 2000$ \\
\hline
\end{tabular}

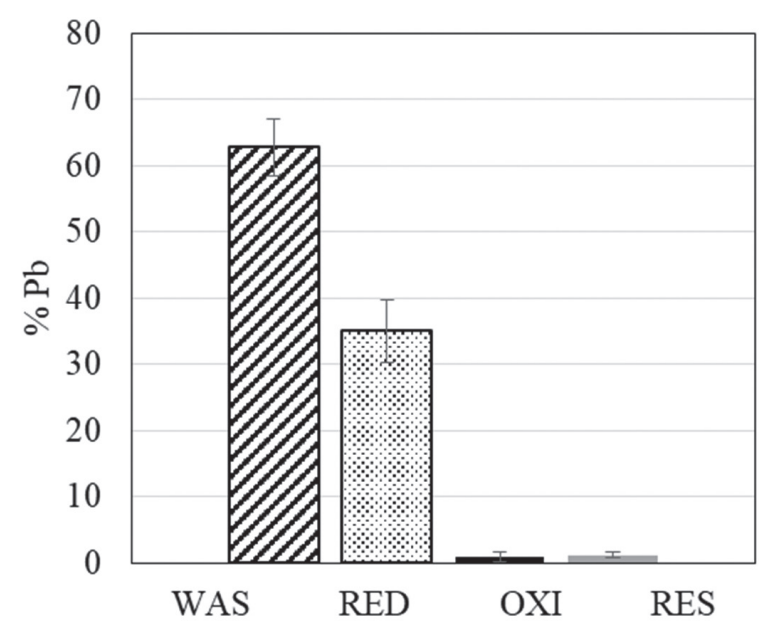

Figura 4. Porcentaje de Pb en cada fracción del método secuencial BCR aplicado al suelo sin tratar

con el metal ligado a materia orgánica y sulfuros y con la fase residual (RES), relacionada con el metal que se encuentra formando parte de la estructura cristalina mineralógica que compone el suelo, fueron despreciables.

\section{Ensayo en tanque agitado}

La evolución del valor de pH de la mezcla suelo-disolución y del porcentaje de $\mathrm{Pb}$ disuelto frente a los moles de $\mathrm{Na}_{2}$ EDTA, expresados como moles de protones por $\mathrm{kg}$ de suelo seco, se presentan en la Figura 5 (a). Como puede observarse, a medida que se añade el agente complejante, $\mathrm{Na}_{2}$ EDTA, el valor del $\mathrm{pH}$ disminuyó hasta un valor de 6.3 y, simultáneamente, el porcentaje de $\mathrm{Pb}$ disuelto aumentó hasta alcanzar un $82 \%$. Estos valores se mantuvieron constantes durante 15 horas, lo que indicó que se había alcanzado el equilibrio.

Con objeto de determinar la especiación del contaminante bajo las condiciones experimentales propuestas, se llevó a cabo la implementación de un modelo matemático sencillo empleando el software Visual Minteq. ${ }^{34}$ Para ello, se definió la matriz sólida a partir de la combinación de varios minerales (cuarzo, hidrocerusita, calcita y magnesita), ${ }^{35}$ teniendo en cuenta la concentración inicial de los metales en el suelo, y se estableció un modelo de especiación geoquímica considerando la concentración final del agente complejante empleado en los ensayos en tanque agitado, $\mathrm{Na}_{2}$ EDTA. De acuerdo a los resultados obtenidos, el $\mathrm{Pb}$ disuelto se encuentra mayoritariamente $(\approx 99 \%)$ como PbEDTA $^{2-}$. Por tanto, tal y como destacaron Yeung et al., se prevé el transporte por electromigración del complejo metal-EDTA con carga negativa hacia el ánodo. ${ }^{27} \mathrm{Con}$ objeto de conocer los cambios en la movilidad del $\mathrm{Pb}$, se muestran los resultados obtenidos al aplicar el método BCR (Figura 5 (b)). 

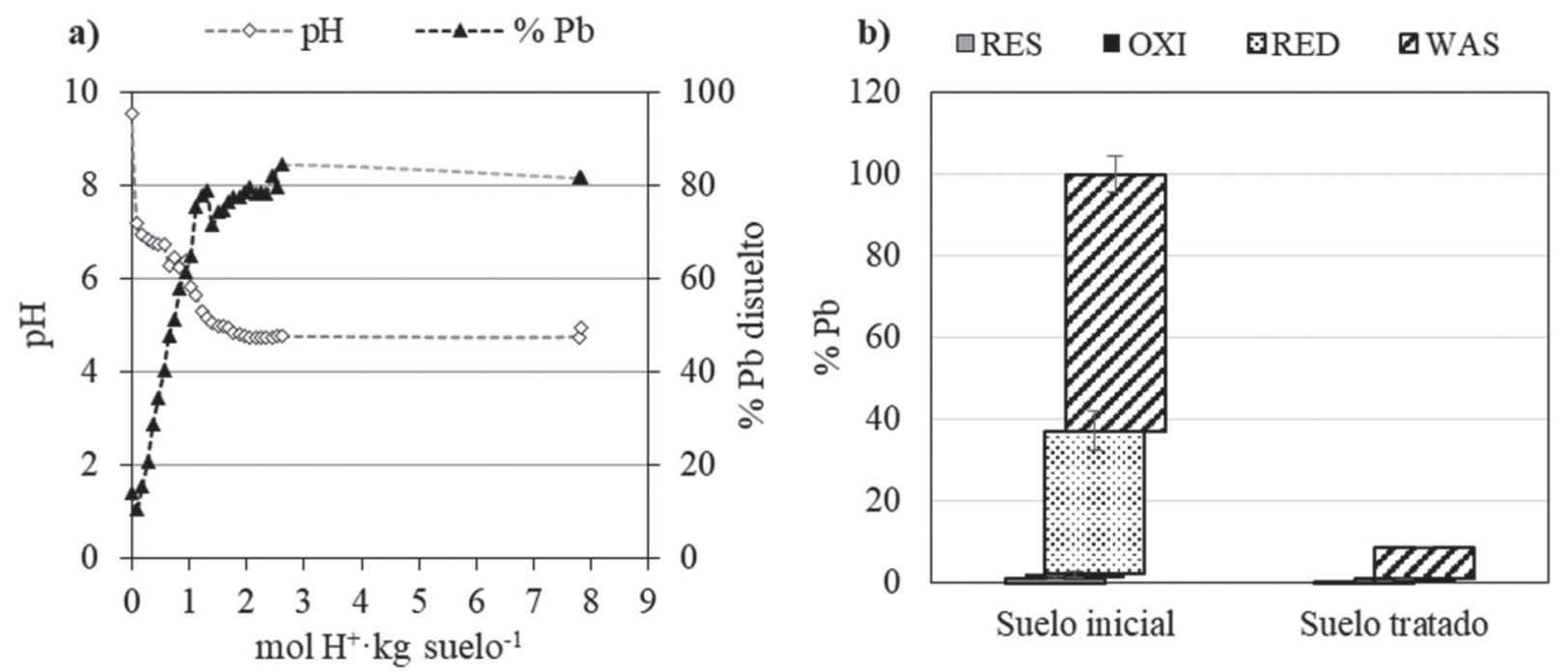

Figura 5. Ensayo en tanque agitado: (a) Evolución del $p H$ y del porcentaje de Pb frente a cantidad de $H^{+}$añadidos. (b) Resultados de aplicar método de extracción secuencial BCR al suelo inicial y tras el ensayo

Como se puede observar, el porcentaje de $\mathrm{Pb}$ en el suelo tras el ensayo en tanque agitado fue de, aproximadamente, un $10 \%$. Estos resultados corroboraron que el $\mathrm{Na}_{2}$ EDTA es una buena opción para ser empleado como agente de mejora del proceso electrocinético en el caso concreto del suelo en estudio.

\section{Ensayos electrocinéticos}

El ensayo I, como se detalla en el Tabla 1, se llevó a cabo con control de $\mathrm{pH}$ a 4 y 8 en el ánodo y cátodo, respectivamente. Durante el experimento, no se detectó plomo en las disoluciones electrolíticas. Con objeto de evaluar si existió movilización de $\mathrm{Pb}$ como consecuencia de la aplicación de corriente, una vez finalizado el ensayo, se aplicó el método BCR al suelo de la columna dividido en dos secciones y se obtuvieron los resultados que se muestran en la Figura 6 (a). De estos resultados se concluye que no existe movilización del contaminante bajo las condiciones experimentales propuestas. Tal y como se describió anteriormente, con objeto de evaluar el transporte por electroósmosis, se lleva a cabo la monitorización de la variación del volumen en los compartimentos electrolíticos externos y de la caída de potencial a lo largo de la columna. Los resultados obtenidos se muestran en la Figura 6 (b).

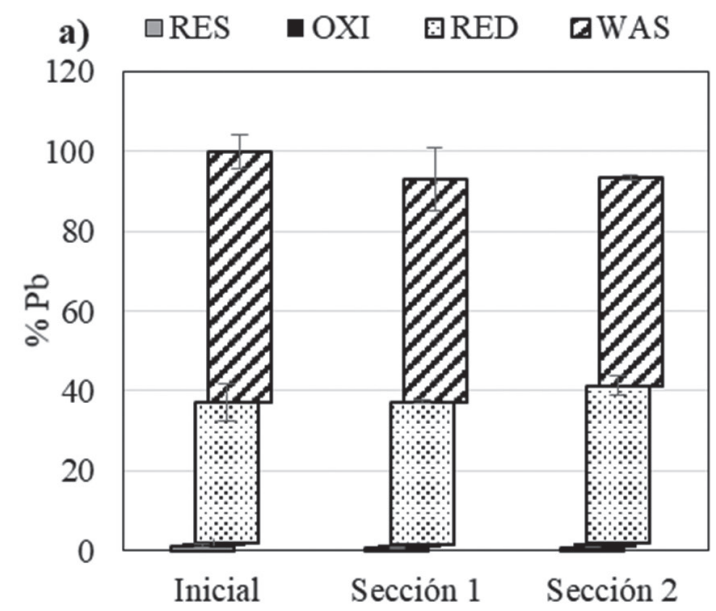

Los resultados muestran que la caída de potencial eléctrico varió a lo largo del tiempo de aplicación de corriente eléctrica. Esto ha sido relacionado con las variaciones de fuerza iónica en la fase acuosa contenida en los poros del suelo. Se debe tener en cuenta que, además de los cambios temporales, la fuerza iónica varía según la posición de la columna. Sin embargo, a partir del ajuste de los datos experimentales a la relación $J_{e o}=k_{e o}^{*}(\Delta V / L)$, se pudo obtener un valor promedio constante de la permeabilidad electroosmótica efectiva de $7.5 \times 10^{-6} \mathrm{~cm}^{2} \mathrm{~V}^{-1} \mathrm{~s}^{-1}$. A partir de los resultados del volumen acumulado en el compartimento catódico, se concluyó que existió un importante flujo electroosmótico desde el ánodo hacia el cátodo.

El diseño del ensayo II pretende aprovechar el flujo electroosmótico detectado en el ensayo I. Para ello, se saturó el suelo inicial con una disolución de $\mathrm{Na}_{2}$ EDTA $10^{-3}$ mol L $\mathrm{L}^{-1}$ con objeto de disolver el $\mathrm{Pb}$ contenido en el suelo. Además, se decidió aumentar el tiempo de duración del experimento. En este caso, se detectó una recuperación del $7 \%$ de $\mathrm{Pb}$ en el cátodo, lo que se relacionó inicialmente con el flujo electroosmótico. Una vez finalizado el ensayo, se llevó a cabo el análisis del suelo aplicando el método normalizado BCR, cuyos resultados se muestran en la Figura 7.

Estos resultados corroboraron la movilización de, aproximadamente, un $10 \%$ del $\mathrm{Pb}$ inicial, lo que es coincidente con

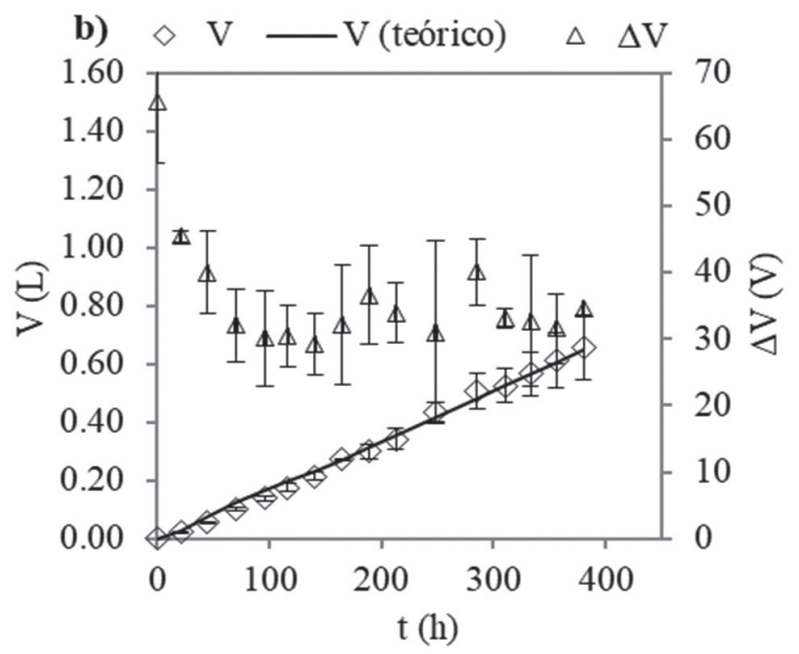

Figura 6. Ensayo I: (a) Resultados de aplicar el método de extracción secuencial BCR al suelo inicial y tras el tratamiento. (b) Evolución del volumen transportado por electroósmosis y del potencial eléctrico y ajuste teórico del flujo electroosmótico considerando constante 


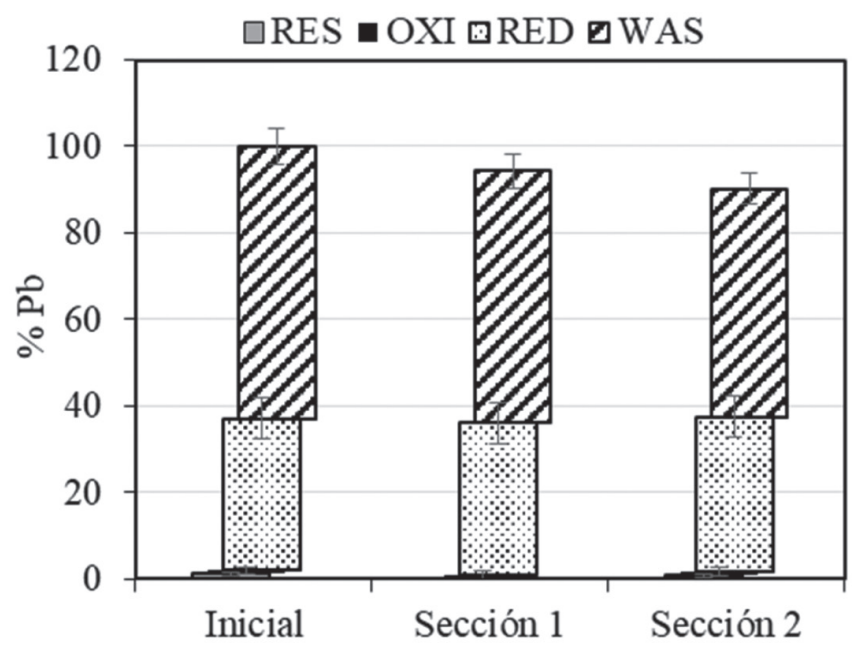

Figura 7. Resultados de aplicar método de extracción secuencial BCR al suelo inicial y tras el ensayo II

la cantidad de metal recuperada en el cátodo por electroósmosis. Con objeto de comprobar la validez de dicha explicación, se llevó a cabo el análisis del flujo electroosmótico siguiendo el procedimiento presentado anteriormente. El valor de permeabilidad electroosmótica efectiva en este caso fue de $6.1 \times 10^{-6} \mathrm{~cm}^{2} \mathrm{~V}^{-1} \mathrm{~s}^{-1}$. Esto confirma que, efectivamente, bajo las nuevas condiciones experimentales, el flujo electroosmótico desde el ánodo hacia el cátodo continuó siendo relevante.

En cualquier caso, la baja recuperación de Pb obtenida para los ensayos I y II, probablemente, esté asociada con la contraposición del flujo electroosmótico desde el ánodo hacia el cátodo y el flujo por electromigración de $\mathrm{PbEDTA}^{2-}$ hacia el ánodo, como se ha reportado en anteriores estudios. ${ }^{28,29}$ Con objeto de aprovechar el flujo electroosmótico en la movilización de Pb, se diseñó el ensayo III cuyas condiciones experimentales se recogen en la Tabla 2. La principal modificación fue el material del que se compone el ánodo, Pt en los ensayos previamente discutidos e hierro para el ensayo III. Varios estudios han propuesto el uso de ánodos de hierro de sacrificio con objeto de controlar los procesos físico-químicos que ocurren como consecuencia del aumento de $\mathrm{pH}$ en el compartimento anódico. Así, Hopkinson et al. (2009) propusieron el suministro de Fe, como agente reductor, desde el ánodo para la estabilización del contaminante presente en el suelo, $\mathrm{Cr}$ (VI). ${ }^{36}$ De esta forma, comprobaron que con

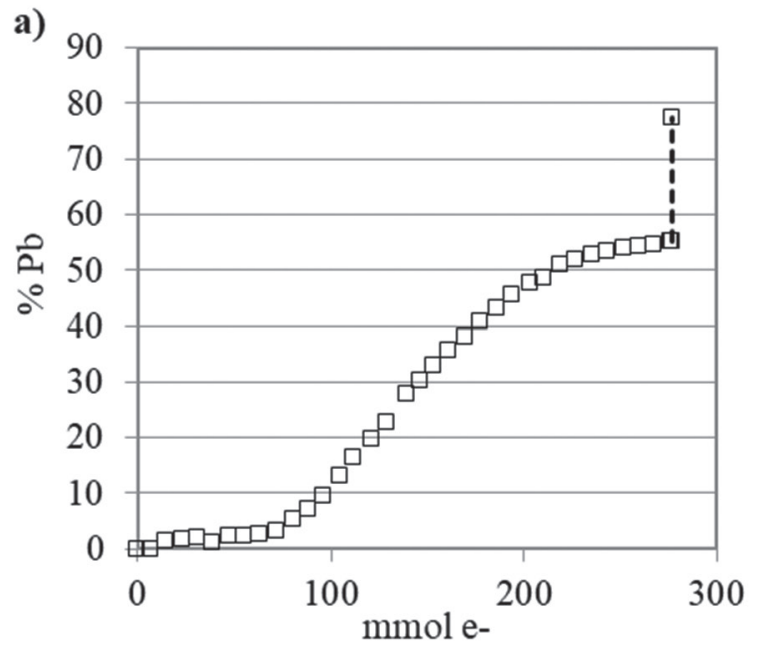

la utilización de un ánodo de sacrificio de Fe se consigue un doble propósito, por un lado, la construcción de barreras físicas y, por otro, la estabilización del contaminante mediante la reducción de $\mathrm{Cr}$ (VI) a $\mathrm{Cr}$ (III) y su posterior precipitación como minerales férricos. Por otro lado, Jeon et al. evaluaron el uso de un ánodo de Fe en un sistema a escala de campo para remediar un suelo contaminado, entre otros metales pesados, con $\mathrm{Pb}$, mediante la técnica electrocinética mejorada con la adición de EDTA en el compartimento catódico. El uso del ánodo de Fe tenía como propósito evitar la acidificación del suelo como resultado de la reacción de oxidación del agua en el ánodo. A partir de los resultados experimentales, se concluyó que tuvo lugar la formación de complejos de carga negativa Pb-EDTA, que se movilizaron hacia el compartimento anódico llegando a un porcentaje de eliminación de, aproximadamente, un 50\% tras 24 semanas de tratamiento. Sin embargo, en este estudio se destaca que las condiciones básicas de $\mathrm{pH}$ en el compartimento anódico produjeron problemas técnicos relacionados con la precipitación de $\mathrm{Fe}$ como $\mathrm{Fe}(\mathrm{OH})_{3}$ en el compartimento anódico. ${ }^{37}$

Con objeto de evaluar el efecto de la incorporación de Fe al anolito en el comportamiento del contaminante, $\mathrm{Pb}$, teniendo en cuenta la presencia de EDTA como agente complejante, se llevó a cabo el ensayo cuyos resultados se presentan a continuación. En el ensayo III, el valor de $\mathrm{pH}$ de control en el compartimento anódico se disminuyó hasta 3.5 para que el Fe estuviese en disolución y, de esta forma, pudiese viajar a través del suelo por electromigración hacia el compartimento catódico. En la Figura 8 (a) se presenta el porcentaje de recuperación de $\mathrm{Pb}$ a lo largo del ensayo. El último punto tiene en cuenta el $\mathrm{Pb}$ en disolución y el que precipitó en el compartimento catódico. Como puede observarse, existió un aumento considerable del porcentaje de $\mathrm{Pb}$ recuperado en el catolito a lo largo del ensayo respecto a los ensayos previos alcanzando casi un $80 \%$. El análisis posterior del suelo mostró una importante disminución de $\mathrm{Pb}$ en el suelo, siendo menor la cantidad de metal en el suelo cercano al ánodo (Figura 8 (b)).

Con objeto de estudiar la importancia del flujo electroosmótico en este ensayo, siguiendo el mismo procedimiento que el expuesto para los ensayos previos, se obtuvo un valor permeabilidad electroosmótica efectiva de $6.4 \times 10^{-6} \mathrm{~cm}^{2} \mathrm{~V}^{-1} \mathrm{~s}^{-1}$, valor del mismo orden de magnitud que los obtenidos en los ensayos I y II. Esto pone de manifiesto que el cambio de condiciones experimentales no tuvo un efecto importante en el flujo electroosmótico.

A la vista de los resultados, se concluye que el cambio en el comportamiento del contaminante se debió a la modificación del valor de $\mathrm{pH}$ en el ánodo junto con el uso de un ánodo de sacrificio. Así,

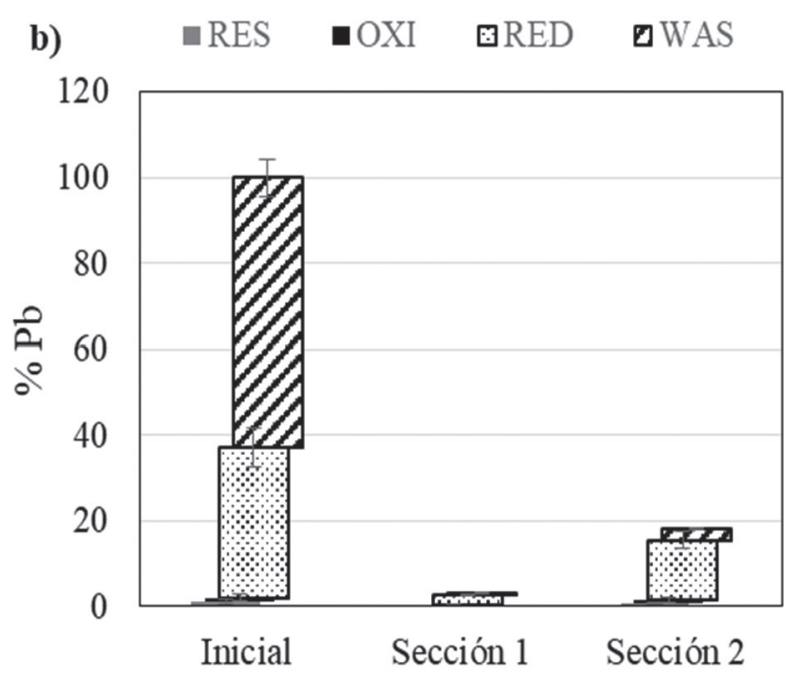

Figura 8. Ensayo III: (a) Evolución de la concentración de Pb recuperado en el catolito durante el tratamiento y (b) Resultados de aplicar método de extracción secuencial BCR al suelo inicial y tras el tratamiento 
el control de $\mathrm{pH}$ a 3.5 permitió que el Fe que provenía del electrodo se encontrase en disolución como $\mathrm{Fe}^{3+}$ (ecuaciones 1 y 2) y viajase desde el ánodo hacia el cátodo por electromigración.

$$
\begin{aligned}
\mathrm{Fe} & \rightarrow \mathrm{Fe}^{2+}+2 \mathrm{e}^{-} \\
\mathrm{Fe}^{2+}+0.25 \mathrm{O}_{2}+\mathrm{H}^{+} & \rightarrow \mathrm{Fe}^{3+}+0.5 \mathrm{H}_{2} \mathrm{O} \\
\mathrm{Fe}^{3+}+3 \mathrm{OH}^{-} & \rightarrow \mathrm{Fe}(\mathrm{OH})_{3}
\end{aligned}
$$

Teniendo en cuenta el carácter básico del suelo, por cada mol de $\mathrm{Fe}^{3+}$ que precipitó como $\mathrm{Fe}(\mathrm{OH})_{3}$ (ecuación 3), tuvo lugar la adición de 3 moles de $\mathrm{H}^{+}$. La disminución de $\mathrm{pH}$ en el suelo, corroborada experimentalmente, facilitó la disolución de $\mathrm{Pb}$ y su posterior transporte por electroósmosis hacia el cátodo. Además, teniendo en cuenta los valores de las constantes de formación de complejos Metal-EDTA para el $\mathrm{Fe}^{3+}$ y el $\mathrm{Pb}^{2+}\left(\log K\left(\mathrm{Fe}(\mathrm{EDTA})^{-}\right)=25 ; \log K\left(\mathrm{~Pb}(\mathrm{EDTA})^{2-}\right)\right.$ $=18.04),{ }^{38}$ se prevé una menor formación del complejo $\mathrm{Pb}(\mathrm{EDTA})^{2-}$ debido a la mayor estabilidad de los complejos Fe-EDTA. Por tanto, se prevé que el contaminante, $\mathrm{Pb}$, bajo las condiciones experimentales propuestas, se encuentre como especie catiónica, $\mathrm{Pb}^{2+}$, lo cual explicaría su recuperación en el cátodo. De esta forma, el movimiento del Pb sería desde el ánodo hacia el cátodo, tal y como corroboran los resultados, debido al transporte por electromigración y por electroósmosis. Estos resultados están de acuerdo con otros estudios en los que se concluye que, por debajo de un valor de $\mathrm{pH}$ igual a 4, el EDTA se encuentra, principalmente, formando complejos con el Fe (III), tales como FeEDTA $^{-}$y FeHEDTA(aq). ${ }^{37}$

Con objeto de comprobar la influencia del uso de un ánodo de sacrificio en el consumo de reactivos, $\mathrm{NaOH}$ y $\mathrm{Na}_{2}$ EDTA, en el control de $\mathrm{pH}$, se calculó la ratio entre el consumo experimental y teórico de estos reactivos. El consumo teórico se obtuvo considerando que la producción de $\mathrm{H}^{+}$y $\mathrm{OH}^{-}$en el ánodo y en el cátodo, respectivamente, se corresponde con la corriente circulada. De esta forma, se obtuvo que la ratio del consumo de $\mathrm{NaOH}$ experimental y teórico fue de 0.73 , es decir, el consumo experimental de $\mathrm{NaOH}$ fue menor que el calculado teóricamente. Esto confirma que, además de la generación de $\mathrm{H}^{+}$por electrólisis del agua, tuvo lugar la oxidación del $\mathrm{Fe}$ contenido en el ánodo, tal y como puede observarse en la Figura 9. Además de la mejora en la recuperación del suelo contaminado, la disminución del consumo de $\mathrm{NaOH}$ en el control de $\mathrm{pH}$ en el ánodo resulta de gran interés desde el punto de vista operativo. En cuanto al consumo de $\mathrm{Na}_{2}$ EDTA, no se observaron diferencias importantes entre el obtenido experimentalmente y el teórico, lo cual refleja que en el compartimento catódico la reacción redox que tuvo lugar fue la electrólisis del agua.

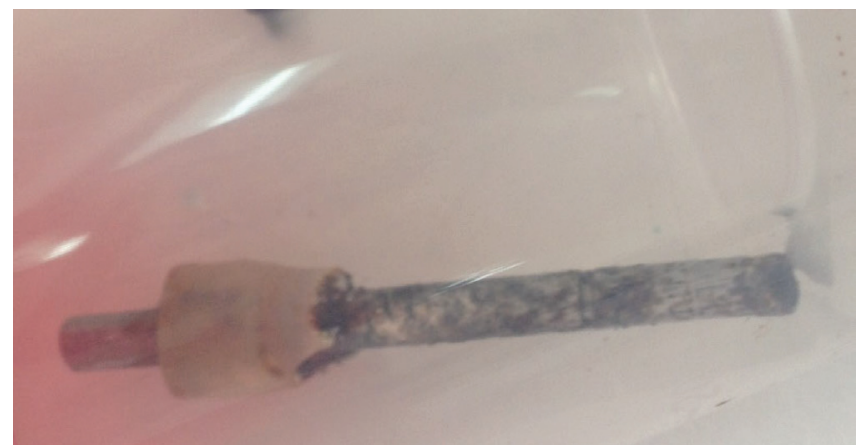

Figura 9. Electrodo de Fe empleado como ánodo tras la aplicación del tratamiento electrocinético

\section{CONCLUSIONES}

A través de la modificación de las condiciones experimentales en los ensayos electrocinéticos mejorados con un agente complejante, se ha llegado a una mayor comprensión de los procesos de transporte y las transformaciones químicas que tienen lugar al aplicar corriente a un suelo real contaminado. Así, a partir de los resultados obtenidos de los ensayos en tanque agitado, se concluye que el agente complejante seleccionado, $\mathrm{Na}_{2}$ EDTA, resulta óptimo para la movilización de plomo en el suelo real estudiado. A pesar de que los estudios preliminares preveían que el $\mathrm{Pb}$ se recuperase por electromigración como $\mathrm{PbEDTA}^{2-}$ en el ánodo, la recuperación del mismo fue despreciable durante los ensayos I y II, llevados a cabo empleando un ánodo de $\mathrm{Pt}$. El transporte neto nulo de $\mathrm{Pb}$ se ha relacionado con el sentido contrapuesto del flujo por electromigración y electroosmótico. El uso de hierro como ánodo de sacrificio permitió la acidificación del suelo mediante la precipitación de $\mathrm{Fe}(\mathrm{OH})_{3}$ y la formación de complejos $\mathrm{Fe}$-EDTA, lo que facilitó la disolución de $\mathrm{Pb}$ y su recuperación en el cátodo mediante transporte por electromigración y electroósmosis. De esta forma, se obtuvieron valores de recuperación de $\mathrm{Pb}$ de, aproximadamente, el $80 \%$ y se prevé una limpieza completa si se aplicase el tratamiento electrocinético durante un periodo de tiempo más largo. Además, cabe destacar que el uso de un ánodo de sacrificio permitió reducir el consumo de $\mathrm{NaOH}$ en el control de $\mathrm{pH}$. Estos resultados prometedores sirven de base para continuar los estudios de optimización de la técnica mejorada con $\mathrm{Na}_{2}$ EDTA/ánodo de sacrificio con objeto de ser aplicada a gran escala.

\section{AGRADECIMIENTOS}

M. Villen-Guzman agradece la Universidad de Málaga la financiación de su contrato postdoctoral. Los autores agradecen la financiación del programa "Proyectos $\mathrm{I}+\mathrm{D}+\mathrm{i}$ en el marco del Programa Operativo FEDER Andalucía 2014-2020", No. UMA18-FEDERJA-279.

\section{REFERENCIAS}

1. Gill, D. A.; Mix, T. L. In An Introduction to Interdisciplinary Toxicology; Pope, C. N., Liu, J., eds.; Academic Press: London, 2002, chap. 25.

2. Alloway, B. J.; Ayres, D. C. In Chemical principles of environmental pollution. Blackie academic \& professional: London, 1987, chap.7.

3. Bradl, H. B.; J. Colloid Interface Sci. 2004, 277, 1.

4. Bacon, J. R.; Davidson, C. M.; Analyst 2008, 133, 25.

5. Filgueiras, A. V.; Lavilla, I.; Bendicho, C; J. Environ. Monit. 2002, 4, 823.

6. Tessier, A.; Campbell, P. G. C.; and Blsson, M.; Anal. Chem. 1979, 51, 844.

7. Rauret, G.; Lopez-Sanchez, J.-F.; Sahuquillo, A.; Barahona, E.; Lachica, M.; Ure, A. M.; Davidson, C. M.; Gomez, A.; Luck, D.; Bacon, J.; J. Environ. Monit. 2002, 2, 228

8. Villen-Guzman, M.; Paz-Garcia, J. M.; Rodriguez-Maroto, J. M.; Gomez-Lahoz, C.; and Garcia-Herruzo, F.; Sep. Sci. Technol. 2014, 49, 1461.

9. Villen-Guzman, M.; Paz-Garcia, J. M.; Rodriguez-Maroto, J. M.; Garcia-Herruzo, F.; Amaya-Santos, G.; Gomez-Lahoz, C.; VeredaAlonso, C.; Electrochimica Acta 2015, 181, 139.

10. García-Rubio, A.; Rodríguez-Maroto, J. M.; Gómez-Lahoz, C.; GarcíaHerruzo, F.; Vereda-Alonso, C.; Electrochimica Acta 2011, 56, 9303.

11. Acar, Y. B.; Environ. Sci. Technol. 1993, 27, 2638.

12. Acar, Y. B.; Alshawabkeh, A. N.; Gale, R. J.; Waste Manage. 1993, 13, 513.

13. Probstein, R. F.; Hicks, R. E.; Science 1993, 260, 498.

14. Shapiro, A. P.; Probstein, R. F.; Environ. Sci. Technol. 1993, 27, 283.

15. Ugaz, A.; Puppala, S.; Gale, R. J.; Acar, Y. B.; Chem. Eng. Commun. 1994, 129, 183 
16. Reddy, K. R.; Cameselle, C. In Overview of Electrochemical Remediation Technologies. In Electrochemical Remediation Technologies for Polluted Soils, Sediments and Groundwater; Reddy, K. R., Cameselle, C., eds.; John Wiley \& Sons Inc: Hoboken, 2009, chap.1.

17. Puppala, S. K.; Alshawabkeh, A. N.; Acar, Y. B.; Gale, R. J.; Bricka, M.; J. Hazard. Mater. 1997, 55, 203.

18. Cameselle, C.; Electrochimica Acta 2015 181, 31.

19. Reddy, K. R., Supraja, C.; J. Environ. Eng. 2004, 130, 442.

20. Pazos, M.; Gouveia, S.; Sanromán, M. A.; Cameselle, C.; J. Environ. Sci. Health - Part Toxic Hazardous Subst. Environ. Eng. 2008, 43, 823.

21. Alshawabkeh, A. N.; Puppala, S. K.; Acar, Y. B.; Gale, R. J.; Bricka, M.; Geotech. Spec. Publ. 1997, 532.

22. Kim, C.; Lee, Y.; Ong, S. K.; Chemosphere 2003, 51, 845.

23. Wong, J. S. H.; Hicks, R. E.; Probstein, R. F.; Electrochem. Decontam. Soil Water. 1997, 55, 61.

24. Song, Y.; Ammami, M.-T.; Benamar, A.; Mezazigh, S.; Wang, H.; Environ. Sci. Pollut. Res. 2016, 23, 10577.

25. Di Palma, L.; Mecozzi, R.; J. Hazard. Mater. 2007, 147, 768.

26. Essington, M. E.; Soil and Water Chemistry: An Integrative Approach, $2^{\text {nd }}$ ed., CRC Press: Boca Raton, 2015.

27. Yeung, T. J. A.; Geotech. Eng. 1996, 122, 666.

28. Reddy, K. R.; Swapna, D.; Saichek, R. E.; J. Environ. Eng. 2004, 130, 1357.
29. Villen-Guzman, M.; Garcia-Rubio, A.; Paz-Garcia, J. M.; RodriguezMaroto, J. M.; Garcia-Herruzo, F.; Vereda-Alonso, C.; Gomez-Lahoz, C.; Electrochimica Acta 2015 181, 82.

30. Martínez López, J.; Llamas Borrajo, J.; De Miguel García, E.; Rey Arrans, J.; Hidalgo Estévez, M. C.; Sáez Castillo, A. J.; Appl. Geochem. 2008, 23, 2324.

31. Villen-Guzman, M.; Garcia-Rubio, A.; Paz-Garcia, J. M.; VeredaAlonso, C.; Gomez-Lahoz, C.; Rodriguez-Maroto, J. M.; Chemosphere 2018, 213, 351 .

32. Decreto 18/2015, de 27 de enero, por el que se aprueba el reglamento que regula el régimen aplicable a los suelos contaminados.

33. Ure, A. M.; Quevauviller, P.; Griepink, B.; Int. J. Environ. Anal. Chem. 1993, 51, 135 .

34. Gustafsson, J.; Visual MINTEQ ver. 3.0 beta, KTH Royal Institute of Technology, Dept. of Land and Water Resources Engineering, 2010.

35. Martínez, J.; Hidalgo, M. C.; Rey, J.; Garrido, J.; Kohfahl, C.; Benavente, J.; Rojas, D. J.; Geochem. Explor. 2016, 162, 62.

36. Hopkinson, L.; Cundy, A.; Faulkner, D.; Hansen, A.; Pollock, R.; Electrochemical Remediation Technologies for Polluted Soils, Sediments and Groundwater, John Wiley \& Sons: Hoboken, 2009, p. 179.

37. Jeon, E.-K.; Jung, J.-M.; Ryu, S.-R.; Baek, K.; Environ. Sci. Pollut. Res. 2015, 22, 15763.

38. Martell, A. E.; Smith, R. M.; Critical Stability Constants: Second Supplement, Springer: New York, 2013. 